\title{
Historia de la Sociedad Mexicana de Trasplantes A.C.: Vigésimo Quinto Aniversario
}

\author{
History of the Mexican Society of Transplants A.C. \\ Twenty-fifth Anniversary
}

\author{
Carmen Yolanda Gracida-Juárez, ${ }^{*}$ Josefina Alberú-Gómez, ${ }^{\ddagger}$ Federico Javier Juárez-de la Cruz ${ }^{\S}$ \\ * Departamento de Trasplantes. Cirugía de Trasplantes. Unidad Médica de Alta Especialidad Siglo XXI. Ciudad de México. \\ ‡ Departamento de Trasplantes. Cirugía de Trasplantes. Instituto Nacional de Ciencias \\ Médicas y de la Nutrición "Salvador Zubirán». Ciudad de México. \\ $\S$ Departamento de Trasplantes. Cirugía de Trasplantes. Unidad Médica de Alta Especialidad No. 71, Torreón, Coahuila. (1985-2015).
}

\section{RESUMEN}

Se relata la historia, en forma descriptiva, de la Sociedad Mexicana de Trasplantes desde sus orígenes, médicos fundadores y los que fungieron como presidentes de las mesas directivas, la historia de la Revista Mexicana de Trasplantes y los diferentes eventos importantes que fueron conformando la Sociedad Mexicana de Trasplantes desde su fundación en 1996 hasta el 2021.

Palabras clave: Historia, Sociedad Mexicana de Trasplantes, fundadores.

Es de antaño conocido que la especie humana, al igual que otras especies, tiene un instinto gregario. Esta tendencia a la agrupación entre quienes comparten similitudes y objetivos persigue el intercambio de ideas y de ayuda mutua (Dr. Antonio de la Torre Bravo). Este mismo impulso estuvo presente desde la concepción de la Sociedad Mexicana de Trasplantes, donde la ayuda mutua no se ha limitado a quienes conforman la sociedad, ha perseguido en todo momento exaltar el conocimiento en los múltiples aspectos en trasplantes con el afán de ofrecer las mejores posibilidades a los pacientes que requieren de estos

\section{ABSTRACT}

The story is told, in a descriptive way, of the Mexican Society of Transplants from its origins, founding physicians and the doctors who served as presidents of the boards of directors, the history of the Revista Mexicana de Trasplantes and the different important events that were shaping the Mexican Society of Transplants that happened from its foundation in 1996 to 2021.

Keywords: History, Mexican Society of Transplants, founders.

procedimientos para poder continuar con una vida en las mejores condiciones posibles en cantidad y calidad de años de vida.

La Sociedad ha tenido y tiene como objetivo contribuir al desarrollo y consolidación de un proyecto con una sólida representatividad nacional donde prevalezcan en todo momento los principios fundamentales de ética, orden, equidad, transparencia y justicia.

Las actividades de la Sociedad Mexicana de Trasplantes A.C. (SMT) se iniciaron el 29 de marzo de 1996, fecha en que fue fundada por la Dra. María del Carmen Yolanda Gracida Juárez y un grupo de 26

Citar como: Gracida-Juárez CY, Alberú-Gómez J, Juárez-de la Cruz FJ. Historia de la Sociedad Mexicana de Trasplantes A.C.: Vigésimo Quinto Aniversario. Rev Mex Traspl. 2021; 10 (1): 5-11. https://dx.doi.org/10.35366/99845 
jóvenes profesionales interesados en el Área de los Trasplantes de Órganos (Tabla 1). ${ }^{1}$

El momento histórico en que se fundó la SMT fue muy importante por el hecho de que se conocía por anécdotas que se realizaban trasplantes renales y que requerían cuidados especiales en áreas físicas que necesitaban de aislamiento. Fue un tiempo en el cual ya se tenían establecidos programas de trasplante renal en el Centro Médico Nacional de México en el Distrito Federal (actual Ciudad de México), sitio donde se había realizado el primer trasplante renal exitoso en el país en el año 1963 por el grupo comandado por el Dr. Manuel Quijano Narezo. ${ }^{2}$ Cuatro años más tarde, en 1967, se iniciaron los programas de trasplante renal en el Centro Médico de Occidente en Guadalajara, Jalisco, y en el Centro Médico del Noreste, en la Ciudad de Monterrey, Nuevo León. Estos tres centros hospitalarios pertenecen al Sistema de Salud Mexicano del Instituto Mexicano del Seguro Social. En 1968, el Instituto Nacional de Nutrición «Salvador Zubirán» inicia su actividad en trasplante renal y de ahí en adelante empiezan a desarrollarse programas en toda la República Mexicana que derivarían en el desarrollo de la ciencia del trasplante en nuestro país en forma progresiva e ininterrumpida. Es por eso que el momento histórico en materia de trasplantes que se vivía en ese entonces, particularmente a partir de los años 80 , impulsara el deseo de la comunidad científica, tanto clínica como quirúrgica, sobre la necesidad de la conformación de una Sociedad de Trasplantes para tener el acceso al intercambio académico, científico y que pudiera además ser una Sociedad donde sus puntos de vista y consejos fueran un baluarte en las decisiones de las autoridades de Salud en materia de Trasplantes y Donación. Por otro lado, se tendría un grupo rector en cuanto al entrenamiento de los médicos jóvenes interesados en los diversos temas del trasplante, actualización en la materia, cursos, relaciones a nivel nacional e internacional con sociedades interesadas en el trasplante, pero lo más importante, la relación de los médicos mexicanos para normar conductas diagnósticas y terapéuticas (consensos), que en ese entonces cada grupo tendría sus abordajes propios basados en el entrenamiento personal, experiencia y publicaciones de sociedades en el extranjero.

Surgió la necesidad, cada vez más evidente, de tener representatividad como Comunidad Científica de Trasplantes. Hasta ese entonces, los trabajos clínicos y quirúrgicos en México se presentaban en foros académicos como los congresos del Instituto Mexicano de Investigaciones Nefrológicas (IMIN) y de la Sociedad Mexicana de Nefrología (SMN), hoy Colegio Mexicano de Nefrólogos, así como en otros congresos de la Asociación Mexicana de Cirugía y de la Asociación Mexicana de Gastroenterología (AMG), teniendo esta última una vasta experiencia de 61 años (fundada en 1935). ${ }^{3}$ De esta manera, surgió La Sociedad Mexicana de Trasplantes, tratando de reunir en foros de Nefrología a los médicos trasplantólogos clínicos y quirúrgicos para conocer su interés por contar con una Sociedad Médica que representara al Área de Trasplantes en México, esta labor fue iniciada y planeada por los Médicos Guillermo Antonio Mondragón Ramírez, José Luis Melchor Ortiz y Raúl Borrego Gaxiola, bajo la dirección de la Dra. María del Carmen Yolanda Gracida Juárez.

Tabla 1: Miembros fundadores de la Sociedad Mexicana de Trasplantes, A.C.

\author{
Dr. Álvaro Abascal Arias \\ Dra. Josefina Alberú Gómez \\ Dr. Raúl Borrego Gaxiola \\ Dr. Moisés Cutiel Calderón Abbo \\ Dr. Héctor Cuellar Cabrera \\ Dr. José Galván Díaz \\ Dr. Ernesto Gómez Hernández \\ Dra. María del Carmen Yolanda Gracida Juárez \\ Dr. Alberto Holm Corzo \\ Dr. Federico Javier Juárez de la Cruz \\ Dr. Eduardo Mancilla Urrea \\ Dr. Enrique Martínez Gutiérrez \\ Dr. José Luis Melchor Ortiz \\ Dra. María Eugenia Miranda Chávez \\ Dr. Guillermo Antonio Mondragón Ramírez
}

\author{
Dr. Simón Ojeda Durán \\ Dr. Rafael Reyes Acevedo \\ Dr. Anastasio Salazar Bañuelos \\ Dr. Armando Soberanes Hernández \\ Dr. Raúl Cuauhtémoc Verdin Vázquez \\ Dr. Andoni Vicente Eguía \\ Dr. Jorge Carlos Ruz Díaz \\ Dr. Baltazar Martínez Navarrete \\ Dr. Gonzalo Valdez Burgos \\ Dr. Rafael Humberto Reyes Bueno \\ Dr. Arturo Espinoza Flores \\ Dr. Andrés López Hernández
}


Fue así como se inició la convocatoria a todos los Profesionales del Trasplante en México, para constituir la Sociedad Mexicana de Trasplantes A.C. El grupo inicial incluyó a 27 miembros fundadores y después de seis reuniones la SMT quedó constituida formalmente en el Salón de Reuniones del Hotel Benidorm en el Distrito Federal el día 29 de marzo de 1996. Se contó con el apoyo invaluable de la Compañía Farmacéutica Sandoz de México, sin el cual no hubiera sido posible la logística de movilizar a los médicos trasplantólogos, que se encontraban en diversos puntos de la República Mexicana y además nos apoyaron para tener una sede para las sesiones previas a la constitución de la SMT. Por decisión unánime de la recién formada SMT, se eligió a la Dra. María del Carmen Yolanda Gracida Juárez como su primer Presidente, otorgándole un tiempo de gestión de dos años por la carga de trabajo que llevaría a cabo durante el inicio de la SMT.

Se emitieron los estatutos de la SMT, los cuales han tenido modificaciones en el periodo de estos 25 años de vida (1996-2021).

Se inauguraron formalmente las actividades académicas de la SMT con el Primer Congreso de Trasplantes en la Ciudad de Acapulco, Guerrero, el día 30 de noviembre de 1997 con 121 asistentes, el cual fue un éxito total y desencadenó el júbilo entre los asistentes para seguir trabajando en pro de la naciente sociedad. Desde sus albores, se llevaron a cabo los congresos anuales y se han incluido temas de trasplante clínico y quirúrgico de todos los órganos y tejidos, así como temas de investigación básica en trasplantes, en especial sobre inmunología de los procesos biológicos del trasplante de órganos.

Desde entonces, ha habido miembros destacados de nuestra sociedad que han contribuido al crecimiento académico de la misma, sin faltar a Congresos y otras actividades académicas de la SMT, y algunos de ellos han ocupado la Presidencia de la Sociedad (Figura 1). La Dra. Carmen Yolanda Gracida Juárez y el Dr. Rafael Reyes Acevedo se han destacado por haber ocupado la Presidencia de la Sociedad de Trasplantes de América Latina y el Caribe (STALyC), lo cual dio un impulso mayor a nuestra sociedad, a todos sus miembros y también permitió mostrar a en el ámbito internacional los trabajos de investigación de médicos mexicanos expertos en trasplantes. En este tenor, es menester destacar que, en virtud del crecimiento en materia de trasplante hepático y del número de profesionales involucrados progresivamente en estos programas, ha sido necesario conformar simposios paralelos en los congresos nacionales sobre trasplante hepático, con la participación entusiasta de un número creciente de cirujanos y clínicos dedicados, destacando la labor que para la consecución de estos simposios ha llevado a cabo el Dr. Mario Vilatobá.

Durante estos años, se implementaron otras actividades académicas como las sesiones mensuales que se presentan tanto en forma presencial por los miembros de la Ciudad de México (antes de la pandemia), como en forma digital, en línea, para que puedan participar los interesados a nivel nacional. Se implementaron diferentes cursos anuales tanto propios como los que necesitaban de nuestro respaldo académico, así como también cursos y talleres. Es importante mencionar que durante estos 25 años de vida de nuestra Sociedad, se han organizado tres congresos conjuntos con la Sociedad de Trasplantes de América Latina y del Caribe (STALyC), teniendo presencia de toda América Latina y de otros países.

Además de los congresos conjuntos, se ha cooperado de manera académica con los cursos de extensión en Trasplantes de la STALyC en Centro América y América del Sur, siendo invaluable la participación de Profesores Mexicanos pertenecientes a la SMT, destacándose la Dra. Josefina Alberú Gómez y el Dr. Rafael Reyes Acevedo.

Muy pronto se vio la necesidad de poder contar con un medio para publicar todas las investigaciones, experiencias clínicas y quirúrgicas en el área de trasplantes y se pensó cómo hacerlo viable y de larga permanencia. Los resúmenes de los congresos se publicaban dentro de los mismos programas, o bien en CD grabados para los eventos. Sin embargo, no se contaba con el respaldo académico y que tuvieran valor curricular. Fue en el año 2001, durante la Presidencia del Dr. Federico Javier Juárez de la Cruz, cuando por primera vez se publicaron el programa del V Congreso Milenio Trasplante Ixtapa 2001 y todos los resúmenes que se presentaron, tanto en cartel como exposición oral, con un Suplemento Especial de la Revista Cirujano General de la Asociación Mexicana de Cirugía General ${ }^{4}$ en donde se publicaron 122 trabajos presentados. Esto no se hubiera logrado de no haber sido por las gestiones exitosas que realizó el Dr. Héctor Faustino Noyola Villalobos, quien en ese momento se desempeñaba como Vocal del Centro de la República Mexicana en la Mesa Directiva 2001 y que en 2006 ocupó la Presidencia de la SMT. Logramos conseguir el apoyo financiero necesario para que se publicara, siendo apoyados por el Editor 


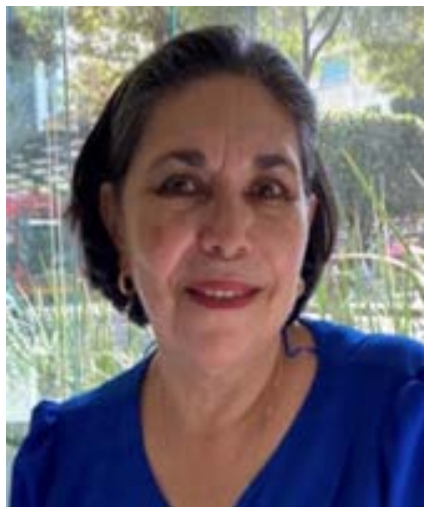

Dra. María del Carmen Yolanda Gracida Juárez

Especialista en Cirugía General Cirugía de Trasplantes (Periodo 1996-1998)

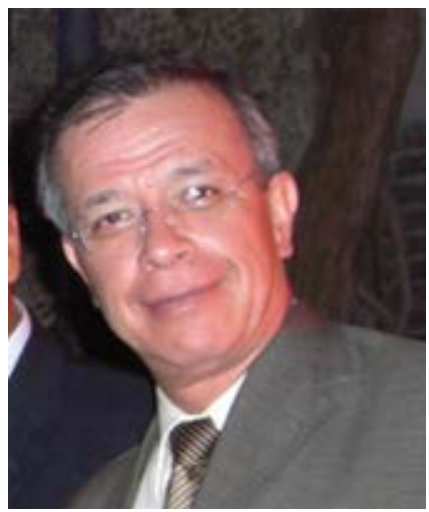

Dr. Francisco Monteón Ramos Especialista en Nefrología, Clínico de Trasplantes (Periodo 2002)

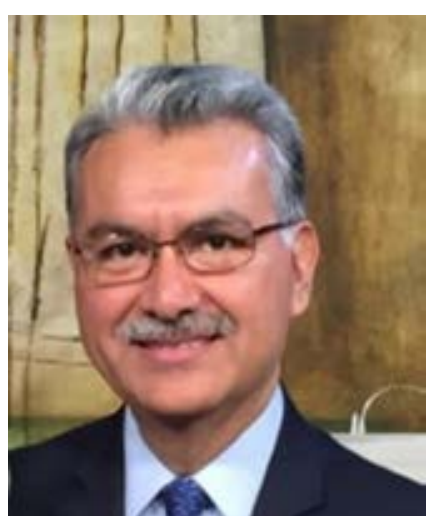

Dr. Enrique Rafael Esparza Chávez

Médico Especialista en Nefrología (Periodo 2006-2007)

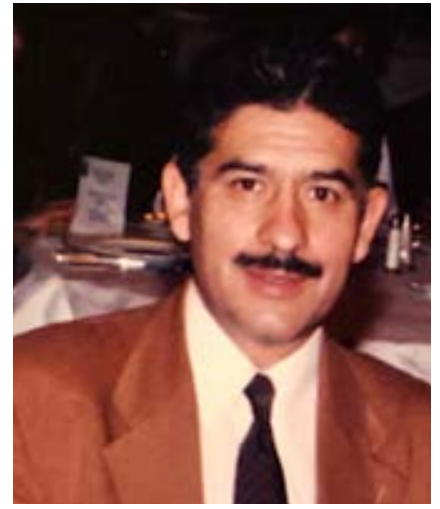

Dr. Guillermo

Mondragón Ramírez

Especialista en Cirugía General Cirujano de Trasplantes (Periodo 1999)

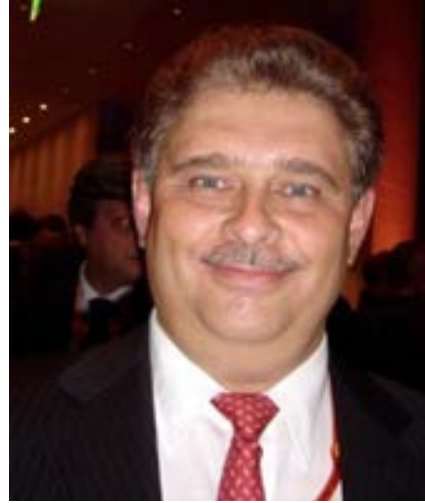

Dr. Alberto Holm Corzo

Especialista en Cirugía General Cirujano de Trasplantes (Periodo 2000)

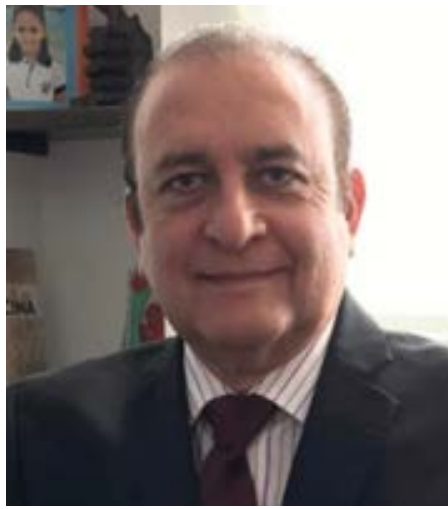

Dr. Federico Javier Juárez de la Cruz

Especialista en Cirugía General Cirujano de Trasplantes (Periodo 2001)

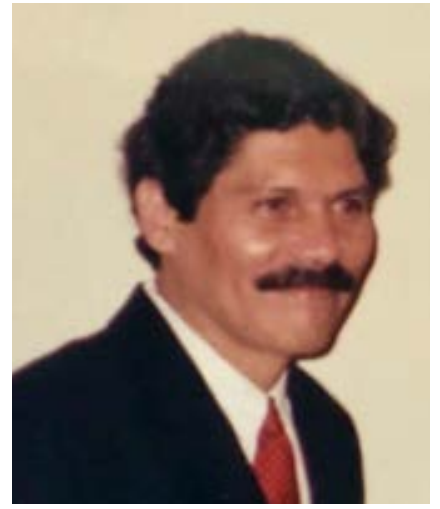

Dr. José Luis Melchor Ortiz Especialista en Medicina Interna Clínico de Trasplante (Periodo 2003)

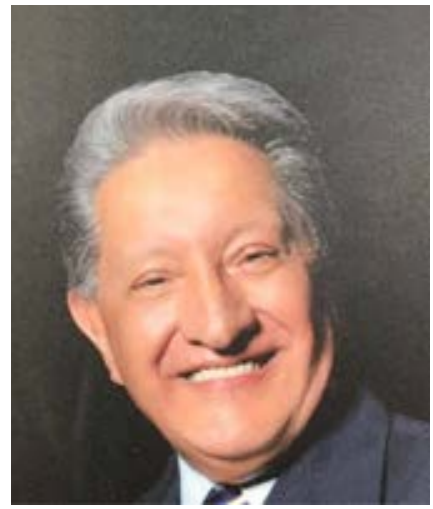

Dr. Simón Ojeda Durán Especialista en Nefrología Pediátrica y Clínico de Trasplante (Periodo 2004)

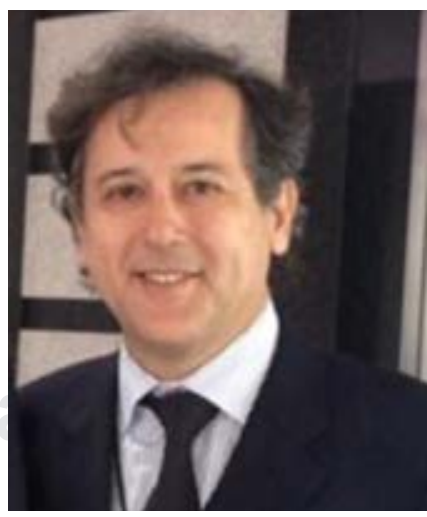

Dr. Federico Mendoza Sánchez Especialista en Cirugía General y Cirujano de Trasplantes (Periodo 2010-2011)

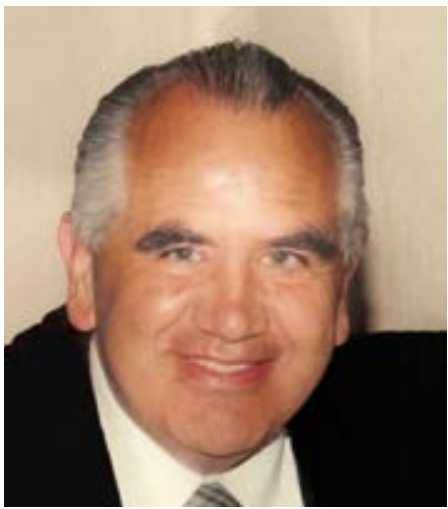

Dr. José Trejo Bellido

Especialista en Cirugía Pediátrica Cirugía de Trasplantes (Periodo 2005)

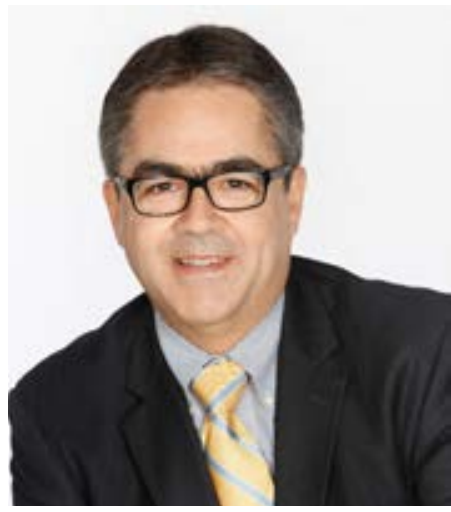

Dr. Rafael Reyes Acevedo

Especialista en Cirugía General y Cirujano de Trasplantes (Periodo 2012-2013)

Figura 1: Presidentes de la Sociedad Mexicana de Trasplantes, A.C 


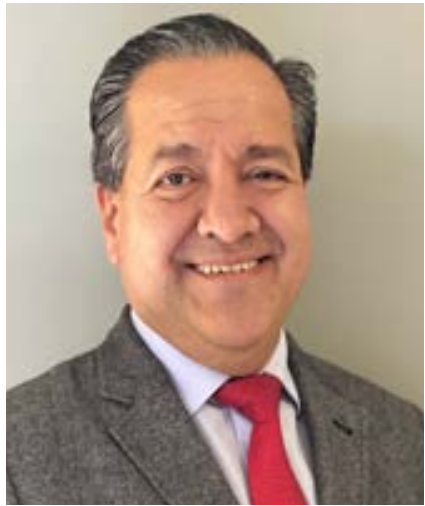

Dr. Luis Eduardo Morales Buenrostro Especialista en Nefrología, Clínico de Trasplantes (Periodo 2014-2015)

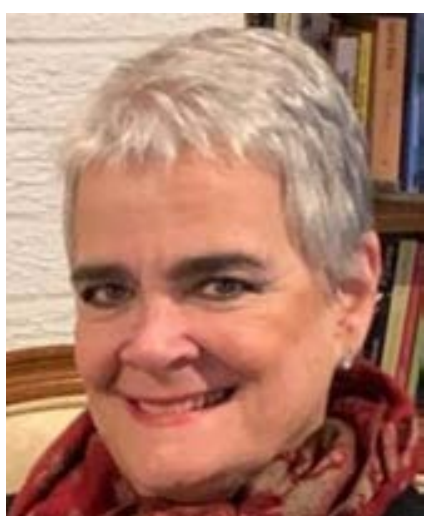

Dra. Josefina Alberú Gómez Especialista en Cirugía General y Cirugía de Trasplantes (Periodo 2018-2019)

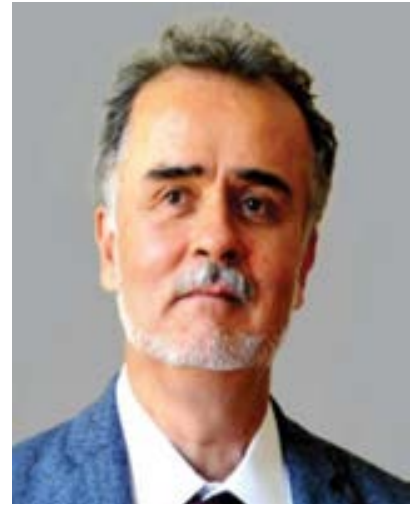

Dr. Benjamín Gómez Navarro Especialista en Nefrología, Clínico de Trasplantes (Periodo 2016-2017)

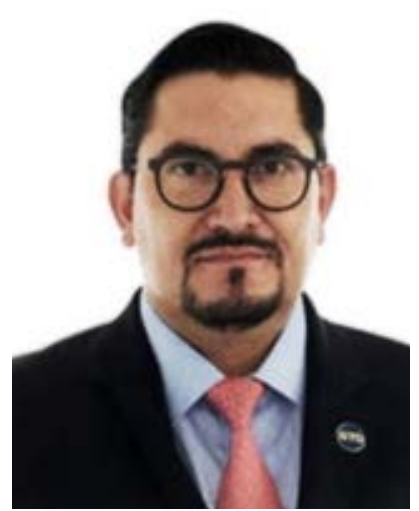

Dr. Rodrigo López Falcony Especialista en Urología y Cirugía de Trasplantes (Periodo 2020-2021)

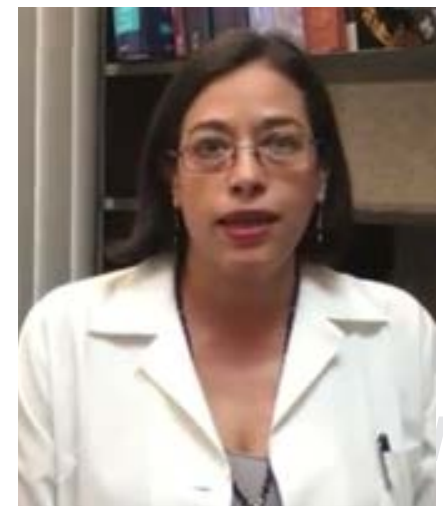

Dra. Mara Medeiros Domingo Especialista en Nefrología Pediátrica Clínica de Trasplantes. Presidenta Electa periodo 2022-2023 de la Revista Cirujano General. El Dr. Guillermo León López, a través de las gestiones del Dr. Héctor Noyola, logró que se contara con valor curricular para esas publicaciones. Por último, 2012 fue el año en que salió a la luz la Revista Mexicana de Trasplantes como publicación cuatrimestral de la SMT. Sólo se publicó la Revista número 1 que correspondía al mes de septiembre-diciembre, dándose a conocer en el XVI Congreso Nacional de Trasplantes en la Ciudad de Guadalajara, Jalisco, durante la Presidencia del Dr. Federico Mendoza Sánchez. ${ }^{5}$ La revista es publicada en formato físico y también en digital en la página de la SMT y se lee en muchos países de América Latina, México incluido desde luego, así como en diferentes partes del mundo, sumando más de 100 países, y su publicación ha sido de manera regular sin faltar un solo número hasta la fecha.

El Dr. Federico Mendoza Sánchez fungió como el primer Editor General de la revista, y el Dr. Gustavo Martínez Mier y el Dr. Luis Eduardo Morales Buenrostro fueron asignados como Editores Asociados en el año 2012. Así continuaron hasta 2014, año en que el Dr. Gustavo Martínez Mier y el Dr. Luis Eduardo Morales Buenrostro fueron nombrados por el Dr. Federico Mendoza Sánchez como Editores Generales y a partir del 2015, durante la Presidencia del Dr. Luis Eduardo Morales Buenrostro, el Dr. Gustavo Martínez Mier fue nombrado como Editor en Jefe, fungiendo como Editores Asociados el propio Dr. Morales Buenrostro y el Dr. Mendoza Sánchez hasta el año 2019. A pesar de los obstáculos se logró la permanencia de la revista y colocarla en el desarrollo y madurez en estos años. Después del año 2020, y con la entrada en la Presidencia del Dr. Rodrigo López Falcony, se nombró Editor en Jefe de la Revista al Dr. Federico Javier Juárez de la Cruz y además se nombraron a la Dra. Josefina Alberú Gómez y a la Dra. Carmen Yolanda Gracida Juárez como Coeditoras, siendo los primeros nombramientos para este puesto de nueva creación.

Se logró la adquisición de una sede fija de nuestra SMT en la Ciudad de México, en el año 2013, con el esfuerzo del Dr. Luis Eduardo Morales Buenrostro y el Dr. Federico Mendoza Sánchez, quienes ocuparon en diferentes periodos la Presidencia de nuestra sociedad, lo cual ha permitido el trabajo de las diferentes mesas directivas y, lo más importante, preservar un archivo histórico de la SMT.

En los últimos dos años la SMT ha sabido adaptarse a las circunstancias que han prevalecido en el ámbito mundial debido a la Pandemia por el virus de 
COVID-19, lo cual ha requerido trabajar a distancia por medio de plataformas electrónicas, manteniendo una asistencia en crecimiento para las sesiones programadas. Cabe destacar que nuestra Revista de Trasplantes fue de las primeras en publicar un suplemento completo acerca del coronavirus (COVID-19) en pacientes con trasplante de órganos. ${ }^{6}$

Se debe destacar el trabajo colaborativo con el Centro Nacional de Trasplantes (CENATRA) y su Director General, el Dr. Salvador Aburto Morales, miembro destacado de nuestra SMT, cuya participación decisiva y formidable en la elaboración de las actividades del XXII Congreso Nacional de la SMT en la Ciudad de Querétaro del 2019 posibilitó que se llevara a cabo el curso TPM (Transplant Procurement Management por sus siglas en inglés) otorgando más de 50 becas completas a diferentes coordinadores de procuración de órganos; en ese mismo año se realizó el curso teórico práctico de toma de botón corneal a cargo de uno de nuestros destacados miembros de la SMT, la Dra. Karla Verdiguel. De manera adicional, un grupo destacado de profesionales en trasplantes desarrolló durante el mismo congreso un curso dirigido a coordinadores de trasplantes con una gran asistencia. Para esa fecha, la Dra. Josefina Alberú Gómez fungía como presidente de nuestra sociedad. Es digno resaltar las reuniones de consenso en donde participaron muchos miembros de nuestra SMT, tal como lo ocurrido con el "Consenso Nacional sobre el estudio de la pareja donante-receptor previo al trasplante renal», que fue publicado en la revista de trasplantes en el $2020,{ }^{7}$ así como las reuniones de consenso realizadas durante 2020 y 2021 para el desarrollo del «Plan de Reactivación de los programas de donación y trasplante» a nivel nacional, organizadas, dirigidas y presididas por CENATRA tras la suspensión de los programas por el impacto que produjo la pandemia por SARS-CoV-2 en las capacidades de las instituciones dedicadas a la práctica de trasplantes y que hubieron de ser convertidas para la atención de pacientes con COVID.

EI CENATRA ha sabido dar a conocer el daño causado por la pandemia de SARS-CoV-2 a pacientes receptores de trasplantes, llevando un registro de la afectación en el ámbito nacional, el cual fue creado ex profeso, con la preponderante participación del Dr. Salvador Aburto Morales y la ardua labor que caracteriza a quienes integran las labores de CENATRA.

Los Congresos se han desarrollado en ciudades, tanto del Norte como del Sur y Centro de la República
Mexicana, destacando la ciudad de Cancún, Quintana Roo en tres ocasiones, Mérida, Yucatán, en dos oportunidades, Guadalajara, Jalisco, en dos ocasiones, Acapulco, Guerrero, en dos, Ixtapa Zihuatanejo en dos, Veracruz, Veracruz, en dos, Aguascalientes, Aguascalientes en una ocasión, Monterrey, Nuevo León, en otra, la Ciudad de Querétaro en una y el último Congreso adaptado a las nuevas circunstancias de manera digital en 2020 en una ocasión.

Los diferentes presidentes han impulsado el aspecto académico de la SMT durante sus periodos de Presidencia, logrando una continuidad y la transición entre las generaciones iniciales y las nuevas, integrando además a coordinadores de trasplante, personal de enfermería, personal médico y paramédicos dedicados a trasplante de órganos y tejidos.

\section{EN CONCLUSIÓN ¿QUIÉNES SOMOS?}

La Sociedad Mexicana de Trasplantes es una Asociación Civil sin fines de lucro que tiene como finalidad lograr una mejor preparación científica, técnica, cultural, moral y social de todos sus asociados, elevar las facultades de los profesionales a fin de ejercer sus labores dentro de un marco ético, con el más alto nivel académico que demanda esta disciplina. Como grupo social que realiza sus actividades para lograr la salud y bienestar del hombre y la sociedad, luchará para que sus miembros actúen con las responsabilidades que esta disciplina impone. Conscientes de que el profesional de trasplantes necesita actualizar sus conocimientos y técnicas en forma permanente, la Sociedad Mexicana de Trasplantes, Asociación Civil, proporcionará ayuda profesional mediante cursos de actualización, conferencias, seminarios, congresos, publicaciones, etcétera.

\section{MISIÓN}

Somos una Sociedad Nacional dedicada de manera prioritaria a la educación médica continua de todos sus socios, con seguridad, honestidad, responsabilidad y compromiso social, en un marco de libertad y lealtad.

\section{VALORES}

Calidad, compromiso social, excelencia, honestidad, humanismo, lealtad, libertad, responsabilidad, seguridad y tolerancia. 


\section{REFERENCIAS}

1. Acta Constitutiva. Sociedad Mexicana de Trasplantes A.C. No. 113,544. Vol. 2974. Ciudad de México. 29 Marzo 1996.

2. Quijano M, Gómez F, Ortiz F, Ronces R. Primeras experiencias de Trasplante Renal en humanos. Gaceta Médica de México. 1964; 94: 93-102.

3. Hurtado $\mathrm{AH}$. Historia de la Asociación Mexicana de Gastroenterología 1935-1991. Ed Dr. Humberto Hurtado Andrade. México, D.F. 1992. pp. 1-623.

4. Cirujano General. México: Graphimedic, S.A. de C.V. Vol. 23 (4) (Supl), 2001. pp. 223-318.
5. Revista Mexicana de Trasplantes. México: Graphimedic, S.A. de C.V. Vol. 1 (1), 2012. pp. 1-42.

6. Revista Mexicana de Trasplantes. México: Graphimedic, S.A. de C.V. Vol. 9 (S2), 2020. pp. s131-s244.

7. Revista Mexicana de Trasplantes. México: Graphimedic, S.A. de C.V. Vol. 9 (S1), 2020. pp. s1-s132.

Correspondencia:

Dra. María del Carmen Yolanda Gracida Juárez E-mail: mcgracida@gmail.com 